\title{
Comparación de cinco métodos para estimar la prevalencia de diabetes mellitus en estudios de base poblacional ${ }^{1}$
}

\author{
Nicanor R. S. Pinto, ${ }^{2,3}$ Laércio J. Franco ${ }^{2,4}$ y José E. C. Moncau ${ }^{2}$
}

RESUMEN Con objeto de aportar información necesaria en la búsqueda de métodos más prácticos y fiables para estudios de base poblacional sobre la diabetes mellitus, en este trabajo se comparan cinco formas de estimar las tasas de prevalencia. Se analizaron datos secundarios de un estudio transversal en una muestra por conglomerados de la población adulta residente en nueve capitales de estados del Brasil, realizado de 1986 a 1988. Los 21846 participantes originales se clasificaron en diabéticos o no diabéticos de acuerdo con cinco métodos distintos: cuestionario domiciliario de toda la población de la muestra (M1), cuestionario individual de la población seleccionada (M2), medición de glucemia capilar en ayunas $\geq 120 \mathrm{mg} / \mathrm{dL}$ (M3), cuestionario individual y glucemia capilar en ayunas $\geq 120 \mathrm{mg} / \mathrm{dL}$ (M4) y cuestionario individual y glucemia capilar en ayunas $\geq 200 \mathrm{mg} / \mathrm{dL}$ y glucemia capilar 2 horas después de sobrecarga oral de glucosa $\geq 200 \mathrm{mg} / \mathrm{dL}$ (M5). Se determinó la concordancia entre los cinco métodos por comparación de las tasas obtenidas y empleo del coeficiente kappa. Las tasas de prevalencia de diabetes estandarizadas por edad variaron según el método analizado; con M1 se subestimaron los valores detectados por M2; con M3 se calcularon valores más altos que con M2 excepto en el grupo de edad de 60 a 69 años, y con M5 las tasas fueron más altas que con M4 excepto en el grupo de edad de 30 a 39 años. Según las tasas estandarizadas por edad, M1 detectó 84\% de los valores estimados por M2; M2 detectó 91\% de los de M3; M3 detectó 70\% de los de M4; y M4 detectó 86\% de los de M5. Las estimaciones de diabetes previamente diagnosticada fueron $64 \%$ y 55\% del total estimado por M4 y M5, respectivamente. Los valores kappa fueron iguales a 0,70 o mayores en M1 contra M2, M1 contra M4, M2 contra M4 y M3 contra M4. Dados los resultados de este estudio, se concluye que los cuestionarios usados en M1 y M2 son métodos apropiados para detectar los casos de diabetes mellitus previamente diagnosticados y se recomienda su uso para evaluaciones o planeamiento de servicios de salud. La medición de glucemia en ayunas (M3) como método exclusivo no reportó ventajas sobre el cuestionario individual (M2). Entre los métodos combinados o múltiples, la glucemia en ayunas junto con el cuestionario individual (M4) fue eficiente en relación con M5, que incorpora la glucemia a las 2 horas después de la ingestión de una sobrecarga oral de glucosa.

1 Basado en "Comparação entre métodos para estimar a prevalência de diabetes mellitus em estudo de base populacional" [tesis de maestría en epidemiología presentada por Nicanor R. S. Pinto a la Universidade Federal de São PauloEscola Paulista de Medicina (UNIFESP-EPM), São Paulo, Brasil, en diciembre de 1993].

2 La correspondencia y solicitudes de separatas deben enviarse a: Nicanor R. S. Pinto, UNIFESP-EPM, Departamento de Medicina Preventiva, Rua Botucatu 740 - V. Clementino, 04023-062, São Paulo, Brasil.Correo electrónico: nicanor@medprev.epm.br
La diabetes mellitus es un síndrome conocido desde hace más de 3000 años, pero solo durante el siglo $X X$ se ha reconocido su verdadera impor-

\footnotetext{
3 Centro de Saúde Coletiva (CeSCo-UNIFESP), São Paulo, Brasil.

4 Centro de Diabetes (CeDi-UNIFESP), São Paulo, Brasil.
}

tancia en la salud de la población. Su magnitud y su impacto como problema emergente de salud pública se han asociado con diversos factores, entre ellos la industrialización, urbanización, aumento de la esperanza de vida, obesidad, vida sedentaria y supervivencia prolongada de los pacientes de diabetes. Si bien actualmente esos factores son similares en casi 
todos los países del mundo, en la mayoría de los países de América Latina, África y Asia hasta hace poco eran escasos los estudios epidemiológicos de enfermedades crónicas no transmisibles, incluida la diabetes mellitus (1).

Los aportes más importantes a la sistematización y consolidación de criterios para el diagnóstico y la clasificación de la diabetes mellitus - de aquí en adelante llamada solo diabetes-, con repercusiones directas en los estudios epidemiológicos de ese síndrome, no se divulgaron hasta principios del decenio de 1980 (2, 3). Hasta ese momento, los resultados de los estudios de prevalencia de diabetes en países de América Latina y el Caribe presentaban variaciones muy amplias $(1,4)$, lo cual hacía difícil compararlos entre sí o con estudios de otras regiones, debido tanto a la dudosa representatividad de las muestras como a los diferentes criterios de diagnóstico aplicados. Actualmente, la comunidad científica internacional tiende cada vez más a aceptar y utilizar los criterios de diagnóstico establecidos por el Grupo de Estudio de la OMS sobre la Diabetes $(3,5,6)$, especialmente en lo que se refiere a estudios de morbilidad y de prevalencia. Además, desde los años ochenta en adelante se ha publicado un buen número de estudios que comparan los diferentes métodos de estimación de la prevalencia de la diabetes (7-14).

La complejidad operacional, la poca aceptación por parte de la población y el costo elevado de la prueba oral de tolerancia a la glucosa justifican la búsqueda de métodos más prácticos para estudios de población, especialmente los de prevalencia y aquellos dirigidos a obtener información para el planeamiento de servicios de salud. Los datos obtenidos anteriormente por el "Estudo Multicêntrico sobre a Prevalência do Diabetes no Brasil" (EMPDB) han permitido comparar distintos métodos para diagnosticar el síndrome en la población brasileña. Nuestro trabajo tiene como objetivo confrontar los resultados obtenidos con cinco métodos distintos para estimar la prevalencia de diabetes en el grupo de edad de
30 a 69 años de una población urbana y analizar la concordancia entre esos métodos.

\section{MATERIALES Y MÉTODOS}

Las características metodológicas del EMPDB aparecen detalladas en un trabajo anterior (15). Por lo tanto, estas se describirán solamente de modo general, pero se referirán los elementos que interesan para evaluar los métodos analizados en este estudio.

\section{Diseño del estudio original (EMPDB)}

El diseño del EMPDB fue de tipo transversal. El estudio se llevó a cabo en una muestra representativa de conglomerados de la población urbana adulta residente en nueve capitales de estados (divisiones político-administrativas) del Brasil. El trabajo original se realizó en tres etapas básicas entre noviembre de 1986 y julio de 1988 (figura 1).

En la primera etapa se identificó a la población objetivo (personas de 30 a 69 años de edad, excluidas las mujeres embarazadas) mediante la administración domiciliaria de un cuestionario a uno de los residentes adultos. Con ese instrumento se clasificó a la población por sexo y edad, por diagnóstico previo de diabetes y por estado de embarazo.

La segunda etapa, limitada a la población elegida, consistió en la administración de un cuestionario a cada persona seleccionada y en la medición y registro de la glucemia capilar en ayunas. El objetivo de esta etapa era seleccionar una submuestra para que participara en la tercera etapa. En función de los valores de la glucemia capilar en ayunas o de que hubiera diagnóstico previo de diabetes, la población de estudio se clasificó en a) pruebas de tamizaje positivas (todas las personas que presentaron glucemia capilar igual a $100 \mathrm{mg} / \mathrm{dL}$ o más, o diagnóstico previo de diabetes) y b) pruebas de tamizaje negativas (aquellos que presentaron glucemia capilar en ayunas menor de $100 \mathrm{mg} / \mathrm{dL}$ y no tenían diagnóstico previo de diabetes).

Todos los participantes clasificados como positivos más una submuestra sistemática de los negativos (que incluyó a todos los individuos situados en sexta posición en un formulario de control de glucemias) fueron invitados a participar en la tercera etapa del estudio. Esta etapa consistió en la administración de un cuestionario más detallado y en la medición de la glucemia capilar a las 2 horas después de haber ingerido una sobrecarga de $75 \mathrm{~g}$ de glucosa. Las dos pruebas de glucemia capilar, en ayunas y 2 horas después de la sobrecarga oral de glucosa, se realizaron en los domicilios de los sujetos entrevistados utilizando tiras reactivas que se leyeron inmediatamente con glucosómetros portátiles, tal como se describe en los resultados generales del EMPDB ya publicados (15). En función de los objetivos del trabajo presente, se reorganizó el banco de datos original del EMPDB y se sometió a un nuevo proceso de consistencia, lo que alteró levemente los totales que aparecen en los trabajos publicados anteriormente $(15,16)$.

\section{Métodos de estimación de la prevalencia de diabetes y definiciones de caso correspondientes}

Definimos como método de estimación de la prevalencia de diabetes al conjunto de procedimientos, técnicas y criterios de clasificación correspondiente a cualquiera de los enfoques aplicados en este estudio. Para calcular la prevalencia de diabetes, se analizaron y compararon dos conjuntos distintos de métodos: 1) métodos con criterios de diagnóstico únicos o exclusivos y 2) métodos con criterios de diagnóstico combinados o múltiples.

\section{Métodos únicos o exclusivos}

Método 1 (M1). Se administró un cuestionario a un adulto residente de cada domicilio para que indicara su conocimiento de personas con diabe- 
FIGURA 1. Diagrama del "Estudo Multicêntrico sobre a Prevalência do Diabetes no Brasil", por etapas, 1986 a 1988

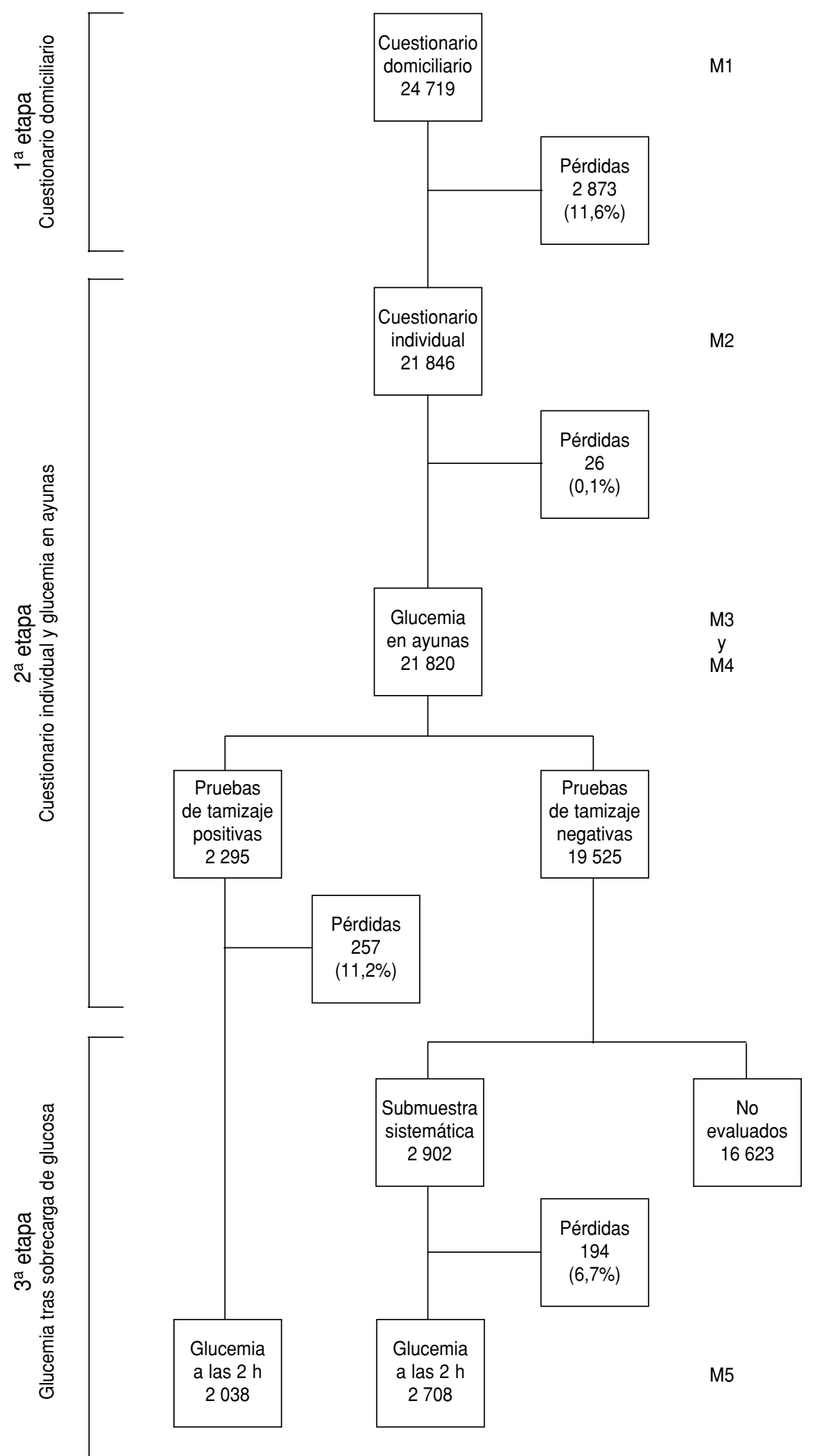

tes entre los residentes del domicilio en particular. Las preguntas principales fueron: "¿Vive aquí alguna persona que tiene diabetes?" y “Quién es?"

Método 2 (M2). Se administró un cuestionario individual que evaluaba el conocimiento del entrevistado sobre un posible diagnóstico previo de diabetes. Las preguntas principales fueron: "¿Está usted enfermo o en tratamiento por alguna enfermedad?" y "Especifique cuál." Si la dolencia mencionada era diabetes, se le preguntaba: "¿Fue un médico quien le dijo a usted que tiene diabetes?"

Método 3 (M3). Se identificaron sujetos con hiperglucemia detectada mediante examen médico. Este método se basó exclusivamente en que la prueba de glucemia capilar en ayunas diera resultados $\geq 120 \mathrm{mg} / \mathrm{dL}$ (5).

\section{Métodos combinados o múltiples}

Método 4 (M4). Se determinó el conocimiento previo de diabetes del entrevistado (M2) y también el valor de glucemia capilar en ayunas $\geq 120 \mathrm{mg} / \mathrm{dL}$ (M3).

Método 5 (M5). Se determinó el conocimiento previo de diabetes del entrevistado (M2) y, además, el valor de glucemia capilar en ayunas $\geq 200 \mathrm{mg} / \mathrm{dL}$ y el valor de glucemia capilar 2 horas después de una sobrecarga oral de $75 \mathrm{~g}$ de glucosa $\geq 200 \mathrm{mg} / \mathrm{dL}$. Este es el método considerado ideal y recomendado por la OMS (5).

Todos los sujetos seleccionados para la segunda etapa del estudio recibieron instrucciones sobre el ayuno y la restricción del uso de fármacos que debían observar la noche anterior a la medición de la glucemia. La OMS recomienda limitar el tiempo de ayuno a entre 10 y 16 horas (5). Sin embargo, en el análisis de nuestro trabajo original (17) no se encontraron diferencias estadísticamente significativas entre las glucemias de los sujetos que habían ayunado el tiempo recomendado por la OMS y las de otros que no se habían ceñido a esa limitación de tiempo. En este trabajo optamos por analizar las glucemias de ayuno sin tener en cuenta esa recomendación de la OMS. 


\section{Técnicas estadísticas}

Las tasas de prevalencia de diabetes obtenidas según los métodos descritos fueron estandarizadas por edad mediante el método directo. Se usó como estándar la población brasileña de 30 a 69 años de edad del censo de 1980 (18). La estimación de la prevalencia por M5 se realizó por medio de la expansión corregida simple de las proporciones observadas entre individuos con resultados positivos e individuos con resultados negativos a las pruebas de la tercera etapa del estudio, en la población de la cual se originaron $(17,19)$.

La muestra de 21820 personas que permitió la clasificación diagnóstica independiente por los métodos M1, M2, M3 y M4 fue también analizada por confrontación directa entre esos métodos, en distintas combinaciones, por medio del coeficiente de concordancia kappa $(20,21)$. Los valores de kappa se interpretaron de la siguiente forma: menores de 0,40, concordancia escasa; de 0,40 a 0,75, concordancia satisfactoria; y mayores de 0,75 , concordancia excelente (20).

\section{RESULTADOS}

En los estudios observacionales basados en poblaciones se prevé la pérdida o abandono de cierto número de participantes y en este estudio ese número aumentó a medida que aumentó también la complejidad de los procedimientos en las diversas etapas (figura 1; cuadro 1).

Las horas de ayuno declaradas por los participantes se aproximaron a una distribución normal con una media de 11,9 horas y una desviación estándar de 2,4 horas. Declararon haber ayunado durante un intervalo de 10 a 16 horas, 18385 sujetos, o sea 84\% del total que participó en esa etapa. Añadiendo 2 horas al intervalo, es decir durante un tiempo total de ayuno de 8 a 16 horas, el número de participantes aumentó a 20 931, o sea 96\% del total.

Según las tasas de prevalencia estandarizadas por edad, M1 detectó $84 \%$ de los valores estimados por M2;

CUADRO 1. Tamaño de las muestras usadas para estimar la prevalencia de diabetes mellitus por cada método evaluado, sexo y grupo de edad en nueve capitales de estados del Brasil, 1986 a 1988

\begin{tabular}{|c|c|c|c|c|}
\hline \multirow{2}{*}{$\begin{array}{c}\text { Grupo de edad } \\
\text { (años) }\end{array}$} & \multicolumn{4}{|c|}{ Tamaño de las muestras } \\
\hline & M1 & $\mathrm{M} 2$ & M3 y M4 & $M 5^{\mathrm{a}}$ \\
\hline \multicolumn{5}{|l|}{ Mujeres } \\
\hline 30-39 & 5418 & 4942 & 4934 & 1036 \\
\hline $40-49$ & 3772 & 3417 & 3416 & 849 \\
\hline $50-59$ & 2900 & 2654 & 2654 & 80 \\
\hline 60-69 & 2055 & 1884 & 1881 & 586 \\
\hline Total (30-69) & 14145 & 12897 & 12885 & 3271 \\
\hline \multicolumn{5}{|l|}{ Hombres } \\
\hline 30-39 & 4152 & 3540 & 3534 & 456 \\
\hline $40-49$ & 2804 & 2357 & 2353 & 373 \\
\hline $50-59$ & 2197 & 1839 & 1837 & 378 \\
\hline 60-69 & 1421 & 1213 & 1211 & 268 \\
\hline Total (30-69) & 10574 & 8949 & 8935 & 1475 \\
\hline \multicolumn{5}{|l|}{ Totales } \\
\hline 30-39 & 9570 & 8482 & 8468 & 1492 \\
\hline $40-49$ & 6576 & 5774 & 5769 & 1222 \\
\hline $50-59$ & 5097 & 4493 & 4491 & 1178 \\
\hline 60-69 & 3476 & 3097 & 3092 & 854 \\
\hline Total (30-69) & 24719 & 21846 & 21820 & 4746 \\
\hline
\end{tabular}

${ }^{\mathrm{a}} \mathrm{M} 5$ = pruebas positivas y pruebas negativas.

M2 detectó 91\% de los valores de M3; M3 detectó $70 \%$ de los de M4; y M4 detectó $86 \%$ de los de M5. Dicho de otra manera, la tasa de prevalencia estandarizada por edad estimada por M5 fue 1,16 veces mayor que la estimada por M4; 1,66 veces mayor que la estimada por M3; 1,82 veces mayor que la estimada por M2; y 2,15 veces mayor que la estimada por M1. Se observó una tendencia similar con las tasas brutas, excepto entre las mujeres cuya prevalencia estimada por M3 fue levemente menor que la estimada por M2 (cuadro 2).

En el cuadro 2 puede observarse también que, según todos los métodos analizados, la prevalencia de diabetes tiende a aumentar con la edad. Se observa, asimismo, la tendencia de las estimaciones de prevalencia a aumentar en los métodos con criterios diagnósticos de mayor complejidad. Las excepciones a esa tendencia corresponden a las mujeres en los grupos de edad de 50 a 59 y de 60 a 69 años en las tasas estimadas por M3 y a los hombres del grupo de 30 a 39 años en las tasas estimadas por M5. En este trabajo no se hará un análisis de esas excepciones, pues rebasaría los límites de estudio propuestos.

La comparación de tasas específicas por edad mostró una tendencia homogénea y regular del método M1 a subestimar los valores obtenidos con M2 en todos los grupos de edad. Con el método M3 se estimaron tasas más altas que con M2, excepto en el grupo de 60 a 69 años; y con M5 se estimaron tasas más altas que con M4, excepto en el grupo de 30 a 39 años (véase el cuadro 2).

En el cuadro 3 pueden apreciarse las concordancias de clasificación diagnóstica por sexo entre los métodos M1, M2, M3 y M4, evaluadas con el coeficiente kappa. Cabe destacar que al confrontar M1 con M2 el valor estimado de kappa pasó de 0,90. En las confrontaciones de M1 con M4, M2 con M4 y M3 con M4, los valores de kappa oscilaron entre 0,69 y 0,87 .

\section{DISCUSIÓN}

En lo que se refiere a la proporción de sujetos que participaron plenamente hasta completar el EMPDB y 
CUADRO 2. Tasas de prevalencia de diabetes mellitus (por 1000 habitantes) por cada método evaluado, sexo y grupo de edad, estandarizadas según la edad, en nueve capitales de estados del Brasil, 1986 a 1988

\begin{tabular}{|c|c|c|c|c|c|}
\hline \multirow{2}{*}{$\begin{array}{l}\text { Grupo de edad } \\
\text { (años) }\end{array}$} & \multicolumn{5}{|c|}{ Tasa de prevalencia } \\
\hline & M1 & M2 & M3 & M4 & M5 \\
\hline \multicolumn{6}{|l|}{ Mujeres } \\
\hline $30-39$ & 9,8 & 14,6 & 18,6 & 28,2 & 28,7 \\
\hline $40-49$ & 23,9 & 30,4 & 32,2 & 48,6 & 50,3 \\
\hline $50-59$ & 63,8 & 73,8 & 73,8 & 110,0 & 120,6 \\
\hline $60-69$ & 104,1 & 117,3 & 94,1 & 151,0 & 186,7 \\
\hline Tasa bruta (30-69) & 38,3 & 46,0 & 44,6 & 68,4 & 76,8 \\
\hline Tasa estandarizada & 37,2 & 44,6 & 43,6 & 66,8 & 74,3 \\
\hline \multicolumn{6}{|l|}{ Hombres } \\
\hline $30-39$ & 6,7 & 7,1 & 19,5 & 21,8 & 16,2 \\
\hline $40-49$ & 20,3 & 22,9 & 35,3 & 42,5 & 59,7 \\
\hline $50-59$ & 52,3 & 60,4 & 66,4 & 90,9 & 125,3 \\
\hline $60-69$ & 63,3 & 71,7 & 79,3 & 109,8 & 147,4 \\
\hline Tasa bruta (30-69) & 27,4 & 30,9 & 41,4 & 53,4 & 66,9 \\
\hline Tasa estandarizada & 26,8 & 30,4 & 40,9 & 52,6 & 66,9 \\
\hline \multicolumn{6}{|l|}{ Totales } \\
\hline 30-39 & 8,5 & 11,4 & 19,0 & 25,5 & 24,6 \\
\hline $40-49$ & 22,3 & 27,4 & 33,4 & 46,1 & 52,8 \\
\hline $50-59$ & 58,7 & 68,3 & 70,8 & 102,2 & 119,2 \\
\hline 60-69 & 87,5 & 99,4 & 88,3 & 134,9 & 171,3 \\
\hline Tasa bruta (30-69) & 33,6 & 39,8 & 43,3 & 62,2 & 70,6 \\
\hline Tasa estandarizada & 32,8 & 38,8 & 42,5 & 60,9 & 70,6 \\
\hline
\end{tabular}

CUADRO 3. Coeficiente kappa ( $K$ ) y desviación estándar de kappa (DE) en la confrontación de concordancia de las tasas estimadas de prevalencia de diabetes mellitus según los métodos M1, M2, M3 y M4, por sexo, en nueve capitales de estados del Brasil, 1986 a 1988

\begin{tabular}{|c|c|c|c|c|c|c|c|}
\hline $\begin{array}{c}\text { Métodos } \\
\text { confrontados }\end{array}$ & $K$ & DE & $Z$ & a & $b$ & $c$ & $d$ \\
\hline \multicolumn{8}{|l|}{ Mujeres } \\
\hline M1 contra M2 & 0,91 & 0,0091 & 100 & 508 & 10 & 84 & 12283 \\
\hline M1 contra M3 & 0,49 & 0,0217 & 22 & 277 & 241 & 298 & 12069 \\
\hline M1 contra M4 & 0,72 & 0,0144 & 50 & 511 & 7 & 370 & 11997 \\
\hline M2 contra M3 & 0,47 & 0,0214 & 22 & 286 & 306 & 289 & 12004 \\
\hline M2 contra M4 & 0,79 & 0,0121 & 66 & 592 & 0 & 289 & 12004 \\
\hline M3 contra M4 & 0,78 & 0,0125 & 62 & 575 & 0 & 306 & 12004 \\
\hline \multicolumn{8}{|l|}{ Hombres } \\
\hline M1 contra M2 & 0,95 & 0,0101 & 94 & 256 & 7 & 20 & 8652 \\
\hline M1 contra M3 & 0,52 & 0,0274 & 19 & 168 & 95 & 202 & 8470 \\
\hline M1 contra M4 & 0,69 & 0,0206 & 33 & 258 & 5 & 219 & 8453 \\
\hline M2 contra M3 & 0,50 & 0,0278 & 18 & 169 & 107 & 201 & 8458 \\
\hline M2 contra M4 & 0,72 & 0,0194 & 37 & 276 & 0 & 201 & 8458 \\
\hline M3 contra M4 & 0,87 & 0,0127 & 68 & 370 & 0 & 107 & 8458 \\
\hline \multicolumn{8}{|l|}{ Totales } \\
\hline M1 contra M2 & 0,92 & 0,0072 & 128 & 764 & 17 & 104 & 20935 \\
\hline M1 contra M3 & 0,50 & 0,0170 & 29 & 445 & 336 & 500 & 20539 \\
\hline M1 contra M4 & 0,70 & 0,0119 & 59 & 769 & 12 & 589 & 20450 \\
\hline M2 contra M3 & 0,49 & 0,0168 & 29 & 455 & 413 & 490 & 20462 \\
\hline M2 contra M4 & 0,76 & 0,0105 & 72 & 868 & 0 & 490 & 20462 \\
\hline M3 contra M4 & 0,81 & 0,0092 & 88 & 945 & 0 & 413 & 20462 \\
\hline
\end{tabular}

$Z$ = Número de desviaciones estándar que kappa se aleja de la media. $a=$ Concordancia en la clasificación de diagnósticos (positivo/positivo).

$\mathrm{b}=$ Discordancia en la clasificación de diagnósticos (positivo/negativo).

$\mathrm{c}=$ Discordancia en la clasificación de diagnósticos (negativo/positivo).

$\mathrm{d}=$ Concordancia en la clasificación de diagnósticos (negativo/negativo) los que se perdieron o lo abandonaron, el estudio brasileño se compara favorablemente con estudios similares llevados a cabo en otros países (2226). Dados los objetivos inmediatos y la magnitud del EMPDB, no se investigó en detalle a los sujetos que no completaron su participación, los cuales se incluyen en la figura 1 como pérdidas. No obstante, sería muy importante estudiarlos para evaluar y calcular la magnitud de ese sesgo de muestreo (27).

En el EMPDB se detectó un pequeño sesgo de selección en cuanto a la proporción de hombres y mujeres que participaron. Hubo más pérdidas entre los hombres $\mathrm{y}$, en consecuencia, una mayor participación relativa de las mujeres en dos ocasiones: en la segunda etapa, comparada con la primera, y en las pruebas negativas de la tercera etapa, comparada con la segunda (15)

Según los datos del EMPDB, las tasas estimadas de prevalencia de diabetes de las mujeres mostraron una tendencia a sobrepasar las de los hombres, sobre todo cuando se calcularon por M1, M2 y M4 (véase el cuadro 2). En los trabajos que han abordado la cuestión de esas diferencias por sexo en poblaciones adultas, se han registrado distintos patrones de la razón entre hombres y mujeres $(1,28)$, lo cual sugiere la influencia de diversos factores en esa relación. Sin embargo, los resultados son congruentes con otros datos del Brasil estimados por métodos similares $(29,30)$ pese a las limitaciones de esos trabajos en cuanto a la representatividad de las muestras y a la estratificación por edad. Es probable que esos datos reflejen en parte la propensión de las mujeres a usar los servicios de salud con mayor frecuencia que los hombres $\mathrm{y}$, por ende, a tener un mejor conocimiento de su estado de salud. En el Brasil esa tendencia se ha notado tanto en estudios sobre la demanda de servicios de salud como en los de base poblacional sobre morbilidad y uso de los servicios $(31,32)$.

Sobre la detección de diabetes en poblaciones asintomáticas, es importante considerar su origen en estudios de cohortes, en los que las concentra- 
ciones elevadas de glucemia se asocian con un mayor riesgo de desarrollar complicaciones vasculares y neurológicas $(33,34)$. Al igual que varias otras medidas biológicas, la glucemia se distribuye a lo largo de un espectro continuo, en una curva unimodal prácticamente simétrica y cercana a la curva normal. Puesto que no hay una separación clara entre la curva de distribución de las glucemias en diabéticos y no diabéticos, la adopción de un punto de corte es una decisión arbitraria, lo cual limita la exactitud o validez de la prueba (35-37).

De los métodos que se usan para detectar la diabetes en poblaciones asintomáticas, el de la glucemia en ayunas se considera el más simple y fisiológicamente apropiado (7, 9-12, 14, 34, 36). No obstante, es un método subutilizado en estudios epidemiológicos de base poblacional, lo que debe atribuirse, por lo menos en parte, a que es difícil confiar en el cumplimiento estricto del ayuno, tal como lo ha expresado el propio Grupo de Estudio de la OMS (5). Aparte de los estudios clásicos (38) y de las recomendaciones de la OMS, el tiempo de ayuno y su influencia en la glucemia no han sido temas específicos de investigaciones orientadas a superar sus posibles limitaciones. Por otro lado, hay ciertos conocimientos sobre el metabolismo de los carbohidratos (39) que podrían aducirse para apoyar una disminución sustancial o por lo menos una reevaluación del tiempo mínimo de ayuno que requiere la prueba.

La importancia de una discusión exclusiva del método M3 es relativa, en la medida en que no se esperaría usarlo aisladamente. No obstante, en el Brasil hay algunas secretarías de salud de estados y municipios que someten a la población adulta a esa prueba en conjunción con campañas de vacunación en masa. Cuando una población ha colaborado sometiéndose a punción digital para la medición de la concentración sanguínea de glucosa, se espera que también colabore respondiendo a un cuestionario específico. En el EMPDB se observó que $21846(88 \%)$ de los individuos seleccionados participaron en la se- gunda etapa, en la que respondieron a un cuestionario. Sin embargo, 26 $(0,1 \%)$ de ellos no quisieron someterse a la prueba de glucemia capilar (véase la figura 1).

Por el análisis del coeficiente kappa se observa que los individuos cuya diabetes se detectó con M3 eran diferentes de los detectados con M2. Esto podría explicarse por el hecho de que en muchos casos la diabetes estaba controlada y la glucemia capilar en ayunas dio resultados menores de $120 \mathrm{mg} / \mathrm{dL}$, o sea, dentro del recorrido normal de valores. Aun así, vale la pena destacar que la proporción detectada por M3 fue muy cercana a la detectada por M2.

Al analizar cuantitativamente las tasas estimadas de prevalencia de diabetes previamente diagnosticada por medio de coeficientes estandarizados por edad para ambos sexos, se detectaron 64 y 55\% por M4 y M5, respectivamente (véase el cuadro 2). La relación entre las estimaciones de diabetes previamente o recién diagnosticadas por M4 y M5 establecida mediante la comparación de coeficientes específicos por edad fue congruente con datos de estudios similares en los que se utilizó la prueba de glucemia tras sobrecarga como método de diagnóstico $(19,40,41)$.

El análisis comparativo de las tasas estimadas de prevalencia de diabetes mediante coeficientes estandarizados según la edad mostró que con M4 se detectó $86 \%$ del total detectado por M5. En la comparación de los dos coeficientes debe resaltarse que, en el grupo de edad de 30 a 39 años, M5 tuvo un desempeño levemente inferior al de M4. Los datos de M5 concuerdan con algunos trabajos que mencionan una tendencia hacia prevalencias más altas de diabetes cuando se mide la glucemia después de una sobrecarga de glucosa en una población asintomática (9). Sin embargo, la estrategia de expansión corregida simple de las proporciones observadas entre individuos con pruebas de tamizaje positivas e individuos con pruebas de tamizaje negativas debe haber contribuido a las pequeñas diferencias detectadas y potenciado el sesgo de selección detectado (15).
Por último, es importante hacer hincapié en que la estandarización de la prueba de glucemia tras sobrecarga oral de glucosa representó un gran avance en el sentido de superar el problema de comparar estudios de diferentes países o regiones de un mismo país con diversos hábitos alimentarios. No obstante, se debe recordar que la prueba tras sobrecarga oral presenta dificultades operativas y ha sido criticada porque puede ser influenciada por la edad, inactividad física, obesidad, dieta, hora del día e infecciones, factores que pueden llevar a sobrestimar la prevalencia de diabetes $(9,11)$.

\section{CONCLUSIONES}

Todos los métodos analizados (M1, M2, M3, M4 y M5) fueron coherentes en que mostraron que en ambos sexos las tasas de diabetes estimadas por grupos de edad tienden a aumentar a medida que avanzan los años. Las tasas de prevalencia de diabetes estandarizadas por edad variaron según cada uno de los cinco métodos analizados, del modo siguiente: M5>M4> $\mathrm{M} 3>\mathrm{M} 2>\mathrm{M} 1$.

Con los métodos M1, M2 y M4, se observaron tasas más altas en las mujeres que en los hombres, lo cual sugiere asociaciones secundarias que este estudio, por su diseño y otras limitaciones, no pudo verificar. Esas diferencias fueron prácticamente inexistentes en las estimaciones de prevalencia por los métodos M3 y M5.

En relación con la diabetes previamente diagnosticada, se observan gran coherencia y concordancia al comparar las estimaciones de prevalencia por M1 y M2. El uso exclusivo de la prueba de glucemia en ayunas (M3) para estimar la prevalencia de diabetes no mostró ventaja alguna en relación con las estimaciones derivadas del cuestionario individual (M2). En cuanto al análisis de los métodos combinados o múltiples, que estiman la prevalencia de diabetes previamente diagnosticada e incorporan la búsqueda de asintomáticos, el método M4 tuvo un buen desempeño en comparación con el M5, 
tanto en las tasas generales como en las específicas por edad y sexo.

\section{Implicaciones y recomendaciones}

Los métodos exclusivos basados en cuestionarios —domiciliario (M1) e individual (M2) - , a pesar de sus limitaciones, deben ser considerados como instrumento para la planificación en salud a nivel regional en función de su bajo costo, facilidad de ejecución, buena aceptación por la población y, en consecuencia, de su bajo potencial para generar sesgos de selección.

El método basado exclusivamente en la glucemia en ayunas (M3) no debería ser utilizado de forma exclusiva. Sin embargo, combinado con el cuestionario individual (M4), resulta un buen método para estudios de carácter regional o incluso nacional pues, comparado con M5, presenta un costo menos elevado, mayor facilidad de ejecución y buena aceptabilidad por la población.

El método M5 debería reservarse para evaluaciones periódicas a largos intervalos, basadas en el análisis de medidas de intervención o comparaciones internacionales, y puede ser incorporado en investigaciones nacionales mediante el muestreo de la población.

Se recomienda realizar otros estudios diseñados específicamente para determinar el potencial de las encuestas domiciliarias en la reevaluación del tiempo de ayuno necesario para un uso más seguro de la prueba de la glucemia en ayunas en estudios epidemiológicos.

\section{AGRADECIMIENTO}

Los autores agradecen las críticas y sugerencias ofrecidas en distintas etapas del manuscrito por Sandra M. Spedo, Waldir L. Alves, Maria Graciela P. Morell, Francisco A. Alves, Clovis A. Peres, Domingos A. Malerbi, Júlio Litivok y Luiz F. Marcopito.

\section{REFERENCIAS}

1. West KM. Epidemiology of diabetes and its vascular lesions. New York: Elsevier; 1978.

2. National Diabetes Data Group - NIH. Classification and diagnosis of diabetes mellitus and other categories of glucose intolerance. Diabetes 1979;28:1039-1057.

3. World Health Organization. Expert Committee on Diabetes Mellitus: second report. Geneva: WHO; 1980. (Informe técnico 646).

4. Organización Panamericana de la Salud. Manual de normas técnicas y administrativas del Programa de Diabetes Mellitus. Washington, DC: OPS; 1984. (Serie PALTEX 2).

5. World Health Organization. Diabetes mellitus: report of a WHO study group. Geneva: WHO; 1985. (Informe técnico 727).

6. Harris MI, Hadden WC, Knowler WC, Bennett $\mathrm{PH}$. International criteria for the diagnosis of diabetes and impaired glucose tolerance. Diabetes Care 1985;8:562-567.

7. Barrett-Connor E. The prevalence of diabetes mellitus in an adult community as determined by history or fasting hyperglycemia. Am J Epidemiol 1980;111:705-712.

8. Woo J, Swaminathan R, Cockram CS, Pang CP, Mak YT, Au SY, et al. The prevalence of diabetes mellitus and an assessment of methods of detection among a community of elderly Chinese in Hong Kong. Diabetologia 1987;30:863-868.

9. Trilling JS. Screening for non-insulindependent diabetes mellitus. Prim Care 1988; 15:285-295.

10. Young TK, Krahn J. Comparison of screening methods in a diabetes prevalence survey among northern Indians. Clin Invest Med 1988;11:380-385.

11. Dowse GK, Zimmet PZ. The prevalence and incidence of non-insulin-dependent diabetes mellitus. En: Alberti KGMM, Mazze RS, eds. Frontiers of diabetes research: current trends in non-insulin-dependent diabetes mellitus. Amster- dam: Excerpta Medica; 1989:37-59. (International congress series 859).

12. Finch $C F$, Zimmet $P$, Alberti KGMM. Determining diabetes prevalence: a rational basis for the use of fasting plasma glucose concentration? Diabetic Med 1990;7:603-610.

13. Cockram CS, Lau JTF, Chan AYW, Woo J, Swaminathan R. Assessment of glucose tolerance test criteria for diagnosis of diabetes in Chinese subjects. Diabetes Care 1992;15: 988-990.

14. Hanson RL, Nelson RG, McCance DR, Beart JA, Charles MA, Pettitt DJ, et al. Comparison of screening tests for non-insulin-dependent diabetes mellitus. Arch Intern Med 1993;153: 2133-2140.

15. Malerbi DA, Franco LJ. Multicenter study of the prevalence of diabetes mellitus and impaired glucose tolerance in the urban Brazilian population aged 30-69 yr. Diabetes Care 1992;15:1509-1516.

16. Bortheiry AL, Malerbi DA, Franco LJ. The ROC curve in the evaluation of fasting capillary blood glucose as a screening test for diabetes and IGT. Diabetes Care 1994;17: 1269-1272.

17. Pinto NRS. Comparação entre métodos para estimar a prevalência de diabetes mellitus em estudo de base populacional [tesis de maestría]. São Paulo: Universidade Federal de São Paulo-Escola Paulista de Medicina; 1993.

18. Fundação Instituto Brasileiro de Geografia e Estatística (FIBGE). Anuário estatístico do Brasil. Rio de Janeiro: FIBGE; 1985.

19. Harris MI, Hadden WC, Knowler WC, Bennett $\mathrm{PH}$. Prevalence of diabetes and impaired glucose tolerance and plasma glucose levels in U.S. population aged 20-74 yr. Diabetes 1987;36:523-534.

20. Cohen J. A coefficient of agreement for nominal scales. Educ Psychol Meas 1960;20:37-46.
21. Fleiss JL. Statistical methods for rates and proportions. 2a ed. New York: John Wiley and Sons; 1981.

22. Sereday MS, Di Toro $\mathrm{CH}$, Correa A, Nusimovich B, Kapeluschnik D. Encuesta de prevalencia de diabetes: metodología y resultados. Bol Oficina Sanit Panam 1979;86:293-305.

23. Mella I, García de los Ríos M, Parker M Covarrubias A. Prevalencia de la diabetes mellitus: una experiencia en grandes ciudades. Bol Oficina Sanit Panam 1983;94:157-165.

24. Forthofer RN. Investigation of nonresponse bias in NHANES II. Am J Epidemiol 1983; 117:507-515.

25. Grupo Encuesta Diabetes La Plata 81. Prevalencia de diabetes en una población urbana argentina. Rev Soc Arg Diabetes 1984;18:20-33.

26. Garancini MP, Calori G, Ruotolo G, Manara E Izzo A, Ebbli E, et al. Prevalence of NIDDM and impaired glucose tolerance in Italy: an OGTT-based population study. Diabetologia 1995;38:306-313.

27. Criqui MH, Barrett-Connor E, Austin M. Differences between respondents and nonrespondents in a population-based cardiovascular disease study. Am J Epidemiol 1978;108: 367-372.

28. Hamman RF. Diabetes in affluent societies. En: Mann JI, Pyorala K, Teuscher A, eds. Diabetes in epidemiological perspective. New York: Churchill Livingstone; 1983:7-42.

29. Lessa I, Almeida FAA, Alves JFA, Souza ME, Jesus MFS, Caricchio R. Prevalência de doenças crônicas em um bairro de Salvador, Bahia, Brasil. Bol Oficina Sanit Panam 1982; 93:376-387.

30. Lebrão ML, Carandina L, Magaldi C. Análise das condições de saúde e de vida da população urbana de Botucatu, São Paulo (Brasil): IV, Morbidade referida em entrevistas domiciliárias, 1983-1984. Rev Saude Publica 1991;25: 452-460. 
31. Radaelli SM, Takeda SMP, Gimeno LID, Wagner MB, Kanter FJ, Mello VM, et al. Demanda de serviço de saúde comunitária na periferia de área metropolitana. Rev Saude Publica 1990;24:232-240.

32. Aquino EML, Menezes GMS, Amoedo MB. Gênero e saúde no Brasil: considerações a partir da Pesquisa Nacional por Amostra de Domicílios. Rev Saude Publica 1992;26: 195-202.

33. Knowler WC, Bennett PH, Hamman RF, Miller M. Diabetes incidence and prevalence in Pima Indians: a 19-fold greater incidence than in Rochester, Minnesota. Am J Epidemiol 1978;108:497-505.

34. Rushforth NB, Miller M, Bennett PH. Fasting and two-hour post-load glucose levels for the diagnosis of diabetes: the relationship between glucose levels and complications of diabetes in the Pima Indians. Diabetologia 1979;16:373-379.

35. Lilienfeld AM. Foundations of epidemiology. New York: Oxford University Press; 1976.

36. Barrett-Connor E. Factors associated with the distribution of fasting plasma glucose in an adult community. Am J Epidemiol 1980;112: 512-523.

37. Zimmet P. Does NIDDM exist? A new look at the classification of diabetes. Int Diabetes Monit 1992;4:1-5.

38. Hayner NS, Kjelsberg MO, Epstein FH, Francis Jr T. Carbohydrate tolerance and diabetes in a total community, Tecumseh, Michigan: 1, Effects of age, sex, and test conditions on onehour glucose tolerance in adults. Diabetes 1965;14:413-423.

39. Dinnen S, Gerich J, Rizza R. Carbohydrate metabolism in non-insulin-dependent dia- betes mellitus. New Engl J Med 1992;327: 707-713.

40. Harris MI. Screening for undiagnosed diabetes: results from the U.S. and Mauritius. Int Diabetes Fed Bull 1990;35:8-10.

41. Dowse GK, Gareeboo H, Zimmet PZ, Alberti KGMM, Tuomilehto J, Fareed D, et al. High prevalence of NIDDM impaired glucose intolerance in Indian, Creole, and Chinese Mauritians. Diabetes 1990;39:390-396.

Manuscrito recibido el 26 septiembre de 1995 y aceptado para publicación en versión revisada el 12 de agosto de 1996.

ABSTRACT To aid in the search for more practical and reliable methods for use in populationbased studies of diabetes mellitus, this article compares five ways of estimating prevalence rates. The analysis was performed on secondary data from a crosssectional study of a cluster sample of the adult population in nine state capitals in Brazil. The original study was carried out from 1986 to 1988 . The 21846 participants were classified as diabetic or not diabetic by five different methods: household questionnaires administered to the entire sample population (M1); individual questionnaires administered to the selected population (M2); measurement of fasting glucose levels in capillary blood, with levels $\geq 120 \mathrm{mg} / \mathrm{dL}$ as the cutoff (M3); individual questionnaire and fasting capillary blood glucose $\geq 120 \mathrm{mg} / \mathrm{dL}$ (M4); and individual questionnaire plus fasting capillary blood glucose $\geq 200 \mathrm{mg} / \mathrm{dL}$ and capillary glucose 2 hours after oral glucose loading $\geq 200 \mathrm{mg} / \mathrm{dL}$ (M5). Agreement between the methods was determined by comparison of the rates obtained and use of the kappa coefficient. The age-adjusted prevalence rates of diabetes varied according to the method used. Values obtained with M1 were lower than those indicated by M2; M3 values were higher than M2 values, except in the age group 60-69 years; and with M5 the rates were higher than with M4, except among persons 30-39 years old. With regard to the age-adjusted rates found by the various methods, M1 detected $84 \%$ of the M2 estimate, M2 detected $91 \%$ of the M3, M3 detected $70 \%$ of the M4, and M4 detected $86 \%$ of the M5. Previously diagnosed diabetes cases accounted for $64 \%$ and $55 \%$ of the totals estimated by M4 and M5, respectively. Kappa values were at least 0.70 for M1 compared against M2, M1 against M4, M2 against M4, and M3 against M4. Based on the results of this study, it was concluded that the questionnaires used in M1 and M2 constituted appropriate methods for detecting previously diagnosed cases of diabetes mellitus, and their use is recommended for the purposes of health services planning or evaluation. Fasting glucose measurement (M3) as the sole method did not show a significant advantage over the individual questionnaire (M2). Of the combined or multiple methods, fasting glucose together with the individual questionnaire (M4) was efficient in comparison to M5, which incorporated measurement of blood glucose 2 hours after oral glucose ingestion. 